\title{
Endurance Running and Its Effect on the Development of Osteoarthritis
}

\author{
James Clatterbuck, Jacob Fridley and Christopher Myers* \\ Department of Health and Human Performance, USA
}

*Corresponding author: Christopher Myers, Department of Health and Human Performance, USA.

Received Date: January 14, 2020

Published Date: January 29, 2020

\begin{abstract}
Endurance running does not cause osteoarthritis. Since endurance running is a very prevalent form of exercise, it is critical to determine the longterm effects that endurance running can have on the body. The hypothesis of this review which is endurance running does not cause osteoarthritis. To come to this conclusion, a multitude of studies relating to this topic were analyzed. Furthermore, the studies analyzed includes variants such as group sizes, species, age groups, and training experience. These studies were utilized to determine the impact of endurance running on the development of osteoarthritis. Additionally, these studies examined many groups that underwent varying lengths of duration, running intensities, and running distances to find a connection between the two factors. A vast majority of the studies examined supported the thesis while only a few sources challenged the thesis. Nevertheless, more research needs to be conducted on this topic to definitively credit or discredit the notion that endurance running does not cause osteoarthritis.
\end{abstract}

Keywords: Endurance; Running; Osteoarthritis; Exercise; Joints

\section{Introduction}

Endurance running is one of the most common modes of exercise for individuals across the globe. It is so widely used because almost anyone can participate in some form of endurance running whether it be a casual jog or marathon running. The health benefits of endurance running are very expansive including weight loss, increasing an individual's VंO2 max, managing anxiety symptoms, reducing symptoms of depression, and so much more. Although endurance running has many positive health benefits, it is natural to wonder what the negative side effects of endurance running are. This wonder especially arises as to how endurance running affects bone and joint health regarding arthritis. Some believe that running adds extra stress on the joints which leads some to think that running plays a role in causing osteoarthritis. The hypothesis of this review which is endurance running does not cause osteoarthritis.

\section{Discussion}

To truly understand if osteoarthritis is caused by endurance running, it is important to comprehend what osteoarthritis truly is. Osteoarthritis is the most common form of arthritis; it is characterized as a degenerative joint disease that involves the cartilage in the joints breaking down Tortora and Derrickson [1]. Cartilage helps to cushion our joints and absorb shock so; the breakdown of cartilage can lead to many unwanted symptoms. Once this breakdown occurs the bones begin to rub together causing pain, discomfort, and joint stiffness. The most common areas that osteoarthritis occurs is in the load-bearing joints, specifically the hips and knees. Once this breakdown occurs, the damage done to the joints becomes irreversible [1]. Due to the irreversible damage of osteoarthritis and its degenerative symptoms, it is important for an endurance runner to understand if osteoarthritis is caused by endurance running. The fact that osteoarthritis tends to affect the load-bearing joints of the hips and knees is especially significant to an endurance runner as these joints are crucial to endurance running regardless of the amount of intensity exerted during exercise.

In a study conducted by Alentorn Geli, et al. [2] they used a systematic review and meta-analysis study design to examine if recreational and competitive running increase chances of osteoarthritis in the knees and hips. An athlete was classified as competitive if they were reported as professional athletes and were 
classified as recreational if they were running in a non-professional manner. Alentorn Geli, et al. [2] examined 25 studies that included 125,810 individuals ranging between professional and recreational runners [2]. Their results showed that recreational runners had a lower chance of developing osteoarthritis compared to competitive runners. The results of this study concluded that running for fifteen or more years greatly increased one's chances for osteoarthritis. Also, the study showed that a sedentary lifestyle also leads to an increased risk of osteoarthritis [2]. This study suggests that prolonged endurance running could cause osteoarthritis but also a sedentary lifestyle could lead to increased risk of osteoarthritis [2]. This could suggest a happy medium of endurance running and that running recreationally could help reduce chances of osteoarthritis as long as it is not excessive or non-existent.

Osteoarthritis tends to affect most individuals later in life. When osteoarthritis does occur, it is normally in those who are above age fifty. Therefore, it is important for researchers to examine an older population when determining if running endurance, the causes an increased chance of osteoarthritis. A study by Lane, et al. [3] did just this. This research examined a group of individuals between the ages of fifty to seventy years old [3]. The goal of this study was to determine if endurance running increased chances of osteoarthritis in older individuals. Of those selected, forty-one were runners and fifty-seven were not runners [3]. All individuals who participated in the study were given an interview, physical examinations, and filled out questionnaires. At the beginning of the study, the runners had a lower mean weight and a lower disability score compared to the non-runners [3]. Radiographs were also taken for both groups to determine the progression of osteoarthritis in the knees, hands, and lumbar spine. The disability scores, weight scores, and radiographs were then reassessed after five years once the study was completed [3]. After the five-year period, both groups had doubled their disability score and the runners still weighed less. Radiographs showed that runners had increased their bone spur score for the knee from 1.3 to 1.5 while non-runners had increased their bone spur score from 0.9 to 1.4 [3]. This study showed that those who ran at an older age had a lower bone spur score and this can suggest that running at an older age does not increase the chances of developing osteoarthritis [3]. The study also showed that increased weight plays a role in osteoarthritis development. This means that endurance running can be used to help manage weight, thus, it could help prevent the development of osteoarthritis [3].

In 2006, Cymet $\mathrm{T}$ and Sinkov V [4], stated that in reasonable amounts, physical activity can reduce the risk of developing certain diseases. However, when the amount of physical activity surpasses the reasonable limit, the protective effect may be offset with an increased risk of injury or osteoarthritis [4]. This was the initial thought; however, this study found that that was not the case [4]. Studies were conducted on both animal subjects and human subjects. In both subject groups, the results presented were insufficient in proving that running was the direct cause of osteoarthritis [4]. Instead, there were other factors that contributed to an increased chance of developing osteoarthritis. These factors are things such as, "A history of injury from overuse or acute trauma as a result of running, excessive running, intrinsic anatomical instability in the joints, or a high body mass index" [4]. Essentially, running itself does not cause osteoarthritis. Instead, it is caused by injuries sustained from running too much, when the body cannot properly support the joint, or if someone is too big/heavy for their frame. All the sources discussed so far have been supportive of this review's hypothesis. However, not all studies found have reported similar results.

While performing a literature review a body of research that supports or challenges the stated thesis, the authors of this review came upon a study that looks at if a gradual strenuous running regimen predisposes to osteoarthritis due to cartilage cell death and altered levels of glycosaminoglycans. In the conclusion of this study conducted by Franciozi CE, et al [5]. The Franciozi CE, et al [5] stated the following: "The articular cartilage of the rats subjected to a gradual strenuous running regimen of controlled intensity exhibited significant predisposing osteoarthritic changes". The conclusions of this study directly challenge the findings of Cymet $\mathrm{T}$ and Sinkov V [4] and the hypothesis for this review. However, one aspect needs to be considered. The Franciozi CE, et al [5] study was performed on rats alone; no humans were tested. The significance of this statement is that there are factors that could have influenced the results. Firstly, rats have a very different lifestyle and diet than humans. Humans have a much more diverse diet which could aid in getting more nutrients that help to protect cartilage in joints. Additionally, humans live a more active lifestyle than lab rats. This additional activity can strengthen and help support human joints. Regardless, the results of this study should be noted; however, this study alone is not enough to discredit the hypothesis. There is one final study found that solidifies the validity of the hypothesis.

The study by Willick SE and Hansen PA [6] is simply titled, "Running and Osteoarthritis." In this study, the authors looked at animal and human models to try to determine if a connection between running and the development of osteoarthritis existed [6]. This study is similar to the other studies cited in this review. However, in this study, the authors also review different studies of the same subject and found similar results to the ones presented in this review. At the end of this study, Willick SE and Hansen PA [6] stated the following: "There is strong evidence that age, prior joint injury, greater body mass index, and heavy manual labor are associated with the development of osteoarthritis". The existing literature does not support an association or causal relationship between low- or moderate-distance running and osteoarthritis" [6]. This finding is significant because the factors that have been found to be associated with osteoarthritis are factors that are often overlooked, leaving running to be the only thing to blame. Every study that has been looked at in this literature review has recommended that if someone wishes to take up the sport of running but has questions or concerns about the impact that it could have on their body, they should consult their physician first. Although most research support the notion that there is no correlation between running and development of osteoarthritis, it 
is still advised that if athletes are finding issues when partaking in the sport of running that they should find an alternate exercise to perform until their injuries are resolved.

\section{Conclusion}

The final results of all these studies support the notion that running does not lead to osteoarthritis. However, there is not an undeniable answer to the notion. The fact that the hypothesis is both supported and challenged calls for the need for more research. Currently, the overwhelming conclusions for various studies state that endurance running does not lead to osteoarthritis. These findings are significant because they could potentially lead to a new point of view on how running affects the human body. These results could open more people up to trying to run or continuing with running if they were on the fence about this exercise modality. The one irrefutable conclusion was that preexisting injuries, anatomical instability, and high body index does lead to higher chances of developing osteoarthritis [4]. Thus, our focus should not be to blame running for osteoarthritis but to look at these other predisposing factors and work on fixing/preventing those.

\section{Acknowledgement}

We would like to thank Kimberly Keyser B.S. for assisting with grammatical corrections to this article.

\section{Conflicts of Interest}

No conflict of interest.

\section{References}

1. Tortora G, Derrickson B (2007) Principles of Anatomy and Physiology $11^{\text {th }}$ (edn). John Wiley and Sons Inc, USA, pp. 284-285.

2. Alentorn Geli E, Samuelsson K, Musahl V, Green CL, Bhandari M, et al. (2017) The association of recreational and competitive running with hip and knee osteoarthritis: a systematic review and meta-analysis. J Orthop Sports Phys Ther 47(6): 373-390.

3. Lane NE, Michel B, Bjorkengren A, Oehlert J, Shi H, et al. (1993) The risk of osteoarthritis with running and aging: a 5-year longitudinal study. J Rheumatol 20(3): 461-468.

4. Cymet T, Sinkov V (2006) Does long-distance running cause osteoarthritis? J AM Osteopath Assoc 106(6): 342-345.

5. Franciozi CE, Tarini VA, Reginato RD, Gonçalves PD, Medeiros VP, et al. (2013) Gradual strenuous running regimen predisposes to osteoarthritis due to cartilage cell death and altered levels of glycosaminoglycans. Osteoarthritis Cartilage 21(7): 965-972.

6. Willick SE, Hansen PA (2010) Running and osteoarthritis. Clin Sports Med 29(3): 417-428. 